\title{
Comparison of Whole Genome Sequencing versus Standard Molecular Diagnostics for Species Identification in the Leishmania Viannia Subgenus
}

\author{
Rachel Lau, ${ }^{1}$ Avinash N. Mukkala, ${ }^{2}$ Ruwandi Kariyawasam, ${ }^{3,4}$ Shareese Clarke, ${ }^{5}$ Braulio M. Valencia, ${ }^{6,7}$ \\ Alejandro Llanos-Cuentas, ${ }^{1,8}$ and Andrea K. Boggild ${ }^{2,9,10 *}$
}

\begin{abstract}
${ }^{1}$ Public Health Ontario Laboratory, Toronto, Ontario, Canada; ${ }^{2}$ Institute of Medical Science, University of Toronto, Toronto, Ontario, Canada; ${ }^{3}$ Division of Diagnostic and Applied Microbiology, Department of Laboratory Medicine and Pathology, University of Alberta, Edmonton, Alberta, Canada; ${ }^{4}$ Alberta Precision Laboratories-Public Health, Edmonton, Alberta, Canada; ${ }^{5}$ Faculty of Nursing, University of Toronto, Toronto, Ontario, Canada; ${ }^{6}$ Kirby Institute, University of New South Wales, Sydney, Australia; ${ }^{7}$ Instituto de Medicina Tropical Alejandro von Humboldt, Universidad Peruana Cayetano Heredia, Lima, Peru; ${ }^{8}$ Facultad de Salud Pública y Administración, Universidad Peruana Cayetano Heredia, Lima, Peru; ${ }^{9}$ Tropical Disease Unit, Toronto General Hospital, Toronto, Canada; ${ }^{10}$ Department of Medicine, University of Toronto, Toronto, Ontario, Canada
\end{abstract}

\begin{abstract}
The prognosis and treatment of New World tegumentary leishmaniasis is dependent on the infecting species, yet such species identification in the Leishmania Viannia subgenus poses a diagnostic challenge. Currently, speciation relies on standard molecular techniques such as restriction fragment length polymorphism (RFLP) analysis, and Sanger sequencing (SS). Whole-genome sequencing (WGS) is a robust and increasingly cost-efficient tool that may improve Leishmania species identification. We evaluated WGS versus standard RFLP-SS for species identification in three reference and five clinical strains of Leishmania Viannia spp. Internal transcribed spacer1 (its1), cysteine proteinase b (cpb), and heat shock protein 70 (hsp70) polymerase chain reaction-restriction fragment length polymorphism (RFLP) was performed, followed by SS of the its2, cpb, hsp70, and mannose phosphate isomerase (mpi) loci. After de novo assembly, sequences were mapped, and homology compared with both reference strains and reference genomes on National Center for Biotechnology Information. All American Type Culture Collection strains were confirmed to be single-species of L. V. braziliensis, L. V. guyanensis, or L. V. panamensis by WGS. Conversely, RFLP-SS was able to definitively identify one of three isolates to the species level. Clinical samples were identified as either single-species $(N=3)$, mixed $(N=1)$, or hybrid $(N=1)$ infections by WGS, while standard molecular diagnosis required multi-target composite analysis for identification due to locidependent results by RFLP-SS. We have corroborated the utility of WGS as a diagnostic tool to speciate members of the L. Viannia subgenus and to discriminate between mixed and hybrid infections. WGS is a potentially useful complement to multistaged RFLP-SS for species identification in Leishmania infections.
\end{abstract}

\section{INTRODUCTION}

The prognosis and treatment of New World tegumentary leishmaniasis is largely dependent on the infecting species. ${ }^{1-3}$ American tegumentary leishmaniasis (ATL) is a subcategorization of cutaneous leishmaniasis (CL) and mucocutaneous (MCL) or mucosal leishmaniasis (ML) of New World origin identified in Central and South America. ${ }^{4}$ ATL exists on a clinical and immunological spectrum, with the different clinical manifestations, treatment responsiveness, and prognoses often attributed to not only host factors but the many infecting species of the Viannia subgenus, including Leishmania Viannia braziliensis, L. V. guyanensis, L. V. panamensis, L. V. peruviana, L. V. lindenbergi, L. V. lainsoni, and L. V. shawi. ${ }^{4}$ In Latin America, for example, it is well recognized that $L$. $V$. braziliensis portends the greatest risk of downstream MCL/ML following CL, while localized CL (LCL) due to $L$. V. panamensis is much more likely to self-cure than some of the other wellrepresented species, such as $L$. V. braziliensis and $L$. V. guyanensis. ${ }^{1-4}$ The presence of mixed or hybrid infections within members of the Viannia subgenus further complicates an already diagnostically challenging situation not only due to the inability to easily discriminate such infections from one another using standard molecular diagnostics ${ }^{5-9}$ but also because of data implicating such hybrids and mixed infections in more complicated disease. ${ }^{10}$

*Address correspondence to Andrea K. Boggild, Tropical Disease Unit, Toronto General Hospital, 200 Elizabeth Street, 13EN-218, Toronto, ON, Canada, M5G2C4. E-mail: andrea.boggild@utoronto. ca
Currently, parasitological methods of diagnosis including culture and Giemsa-stained microscopy remain the gold standard in $\mathrm{ATL}^{11,12}$; however, these techniques are unable to differentiate among members of the Viannia subgenus, which can affect patient treatment, long-term risk stratification, and prognosis. Microscopic examination of Giemsa-stained biopsy smears, aspirates, scrapings, or lesion impressions is further limited by poor sensitivity. ${ }^{11}$ Although culture of tissue specimens is highly specific, poor diagnostic sensitivity, prolonged turnaround time, and required technical expertise limits widespread utility of culture-based diagnosis in ATL. ${ }^{5}$ Although positive cultures provide a rich source of DNA for species identification, such species-level discrimination requires further downstream testing, amounting to a multistaged diagnostic approach. Thus, as with all areas of microbiology, rapid, highly sensitive, and specific molecular methods, including polymerase chain reaction (PCR)-based techniques that are ideally performed on the primary specimen, are quickly supplanting parasitological methods for diagnosis and species identification in ATL. PCR-based methods improve diagnostic sensitivity in specimens with low parasite load and can be used on a wide array of specimens including noninvasively collected filter paper lesion impressions $^{6,7,13}$ and cytology brushes, ${ }^{8}$ targeting 18S rRNA and kinetoplast DNA for confirmation. PCR-based methods including restriction fragment length polymorphism (RFLP) and Sanger sequencing (SS) have been evaluated for species identification, with targets including internal transcribed spacer1 (its1), internal transcribed spacer 2 (its2), heat shock protein 70 ( $h s p 70)$, cysteine proteinase b (cpb), and mannose phosphate isomerase (mpi) all contributing to the molecular diagnostic approach, even in mixed and hybrid infections in 
patients with ATL. ${ }^{7,14-19}$ /ts 1, glycoprotein 63 (gp63), cpb, and hsp70 PCR-RFLP, and its2 and mpi SS have all been shown to identify different members of the Viannia subgenus, but each target has limitations that necessitate the use of multiple amplified and sequenced loci to assign a final species identity. ${ }^{7,18,20-25}$ Despite such multitargeted speciation, there is a rising issue of species misidentification with such a complex approach, as documented in a comparative study of 16 laboratories across Europe and Asia. ${ }^{26,27}$ Thus, there is a dire need for improved approaches to species identification in Leishmania Viannia infections not just for accurate treatment allocation and prognosis but also to inform the molecular epidemiology of leishmaniasis in Latin America.

Non-PCR- or -sequencing-based detection assays, such as multilocus enzyme electrophoresis (MLEE) and MALDI-TOF mass spectroscopy, are alternate methods that have emerged over the past decade, but they are limited to regions with substantial laboratory infrastructure, technical expertise, large volumes of cultured material, and funding for platform maintenance and consumables. ${ }^{10,28,29}$ Whole-genome sequencing (WGS), on the other hand, is a robust and increasingly costefficient molecular tool that may improve Leishmania spp. identification, particularly in scenarios where single target sequences are conserved across closely related species, necessitating sequencing of multiple targets.

In this study, we evaluated WGS as a potential complement to the standard molecular assays, PCR-RFLP and SS, of $c p b$, hsp70, its 1, its2, and mpi loci in a cohort of three reference and five clinical strains of the Viannia subgenus to provide confirmatory species identification. Further, we assessed whether WGS could differentiate potential mixed and hybrid infections within the Viannia subgenus.

\section{METHODS}

Clinical specimens and reference strains. Five primary clinical specimens submitted to the Public Health Ontario Laboratory for Leishmania spp. diagnostic testing that were positive for Viannia spp. and culture positive were included in this analysis. The following $\mathrm{WHO}$ reference strains were obtained from the American Type Culture Collection (ATCC, Manassas, VA) and used as reference strains: $L$. V. braziliensis ATCC 50135 (MHOM/BR/75/M2903), L. V. guyanensis ATCC $50126^{\mathrm{TM}}$ (MHOM/BR/75/M4147), and L. $V$. panamensis ATCC $50158^{\mathrm{TM}}$ (MHOM/PA/71/LS94). ATCC strains and clinical specimens were denoted as Day 0 and Passage 0 upon receipt in our laboratory (S1 Figure).

Leishmania cultures. Clinical and ATCC cultures of Leishmania spp. promastigotes were cultured in Tobie's diphasic culture medium consisting of a blood agar $(0.24 \%$ Laboratory-Lemco Powder, Oxoid; 0.5\% Bacto Peptone, Difco; $1.5 \%$ Bacto Agar, Difco; $0.8 \%$ sodium chloride; and $340 \mathrm{~mL}$ of whole horse blood in a total of $1 \mathrm{~L}$ ) with a liquid overlay $(0.8 \%$ sodium chloride; $0.02 \%$ potassium chloride; $0.02 \%$ calcium chloride; $0.03 \%$ monopotassium phosphate; $0.25 \%$ dextrose; and 100 units $/ \mathrm{mL}$ of penicillin and $100 \mu \mathrm{g} / \mathrm{mL}$ of streptomycin (ThermoFisher Scientific, Waltham, MA) in T25 culture flasks (Nunc, ThermoFisher Scientific) and incubated at 22 to $26^{\circ} \mathrm{C}$ under standard atmospheric conditions. Cultures were propagated until they reached at least 50 million promastigotes ("Baseline") to allow extraction of sufficient DNA $(5 \mu \mathrm{g})$ for WGS. For clinical samples, this could take 40 to 82 days from primary specimen. Promastigotes were collected at two time points 6 weeks apart for analysis (Supplemental Figure 1). An interval period of 6 weeks was chosen to allow sufficient time for promastigotes to achieve the required number of cells for the next DNA extraction (Supplemental Figure 1). All samples were analyzed at two time points except for clinical isolate \#5, which was analyzed at only one time point.

Leishmania cultures were centrifuged at $800 \mathrm{~g}$ for $5 \mathrm{~min}$, following which most of the supernatant was aspirated out, leaving $\approx 1 \mathrm{~mL}$ residual, which was stored at $-80^{\circ} \mathrm{C}$ until DNA extraction.

DNA extraction. DNA was extracted from cultured samples using the DNA mini kit (Qiagen, Germantown, MD) with slight modification from the manufacturer's protocol as follows: cells were lysed at $56^{\circ} \mathrm{C}$ for $1 \mathrm{~h}$; mixing with pipette rather than vortexing after the lysis step to avoid shearing of DNA; DNA was eluted with $60 \mu \mathrm{L}$ of nuclease free water and quantified by Qubit 2.0 fluorometer with broad range dsDNA kit (ThermoFisher Scientific) and $260 / 280$ ratio by Nanodrop ND-1000 spectrophotometer (ThermoFisher Scientific).

PCR and RFLP assays. its1 PCR was performed on DNA extracted from cultured isolates using $370 \mathrm{nM}$ of primers LITSR and L5.8S 20,21 and $3 \mu L$ of DNA in a final volume of $27 \mu \mathrm{L}$ with proofreading polymerase Accuprime Pfx Supermix (ThermoFisher Scientific). Cycling conditions were $95^{\circ} \mathrm{C}$ for 5 min followed by 38 cycles of $95^{\circ} \mathrm{C}$ for $15 \mathrm{~s}, 52^{\circ} \mathrm{C}$ for $30 \mathrm{~s}$, and $68^{\circ} \mathrm{C}$ for $1 \mathrm{~min}$ followed by a final extension of $68^{\circ} \mathrm{C}$ for 5 min in Veriti Thermal Cycler (Applied Biosystems, Waltham, MA). After confirmation of amplification indicated by a $\approx 350$ bp band on $1 \%$ agarose gel, 10 to $15 \mu \mathrm{L}$ of PCR product was digested in $1 \times$ FastDigest Green Buffer and $1 \mu \mathrm{L}$ of Haelll FastDigest enzyme (Thermo Scientific) in a final volume of 30 $\mu \mathrm{L}$ for $1 \mathrm{~h}$ to overnight at $37^{\circ} \mathrm{C}$. Digested PCR products were resolved in $2 \%$ agarose gel in $1 \times$ Tris-borate-EDTA (TBE) and run at $80 \mathrm{~V}$ for $2 \mathrm{~h}$ in the presence of ethidium bromide. its2 PCR was performed with Accuprime Pfx Supermix and $370 \mathrm{nM}$ each of primers LGITSF2 and R2. ${ }^{18,30}$ Cycling conditions for its 2 were $95^{\circ} \mathrm{C}$ for 5 min followed by 45 cycles at $95^{\circ} \mathrm{C}$ for $15 \mathrm{~s}, 60^{\circ} \mathrm{C}$ for $30 \mathrm{~s}$, extension at $68^{\circ} \mathrm{C}$ for $30 \mathrm{~s}$ and a final extension step of $68^{\circ} \mathrm{C}$ for $5 \mathrm{~min}$.

$C p b$ and $h s p 70$ PCR was performed using $370 \mathrm{nM} \mathrm{cpb}$ fwd and rev and hsp70 fwd and rev primers, respectively. ${ }^{7,25,31}$ PCR conditions were the same as its2 except the extension step for each cycle was 90 s instead of 30 s. PCR product (10-15 $\mu \mathrm{L}$ ) was digested in $1 \times$ FastDigest Green Buffer and $1 \mu \mathrm{L}$ of Taql FastDigest enzyme for $c p b$ and Haell FastDigest enzyme for $h s p 70$ (Thermo Scientific) in a final volume of $30 \mu \mathrm{L}$ for $1 \mathrm{~h}$ to overnight at $65^{\circ} \mathrm{C}$ (Taql) and $37^{\circ} \mathrm{C}$ (HaellI), respectively, and 10 to $15 \mu \mathrm{L}$ of $h s p 70$ PCR product was digested in $0.5 \mu \mathrm{L}$ of $\mathrm{Bccl}$ and $1 \times$ CutSmart buffer, in a final volume of $30 \mu \mathrm{L}$ at $37^{\circ} \mathrm{C}$ overnight (New England Biolabs, Ipswich, MA). Digested PCR products were resolved in $2.5 \%$ agarose gel in $1 \times$ TBE and run at $80 \mathrm{~V}$ for $1.5 \mathrm{~h}$ with ethidium bromide. Two PCRs targeting mpi gene were performed using Accuprime Pfx Supermix, $370 \mathrm{nM}$ of each primer and $3 \mu \mathrm{L}$ of DNA in a final volume of $27 \mu \mathrm{L}$. PCR specific to $L$. V. peruviana was amplified with pan-Viannia fwd and $L$. V. peruviana specific rev primers; PCR specific to the other Viannia species was amplified with $370 \mathrm{nM}$ of pan-Viannia fwd and rev primers. $^{7}$ 
Cycling conditions were $95^{\circ} \mathrm{C}$ for 5 min followed by 40 cycles of $95^{\circ} \mathrm{C}$ for $15 \mathrm{~s}$, and $68^{\circ} \mathrm{C}$ for $45 \mathrm{~s}$, with a final extension of $68^{\circ} \mathrm{C}$ for 5 min in Veriti Thermal Cycler (Applied Biosystems). Ten microliters of PCR product were run in 1\% agarose gel in $1 \times$ TAE with ethidium bromide at $100 \mathrm{~V}$ for $30 \mathrm{~min}$ for confirmation.

SS and National Center for Biotechnology Information Basic Local Alignment Search Tool. SS was performed for its2, cpb, hsp70, and mpi regions using $1 \mu \mathrm{L}$ of PCR product (diluted in nuclease free water depending on band intensity) with $1.5 \times$ sequencing buffer, $0.25 \mu \mathrm{L}$ BigDye Cycle Sequencing $v 3.1$, and $1,000 \mathrm{nM}$ primer in a total volume of $10 \mu \mathrm{L}$ (ThermoFisher Scientific) for both forward and reverse primers. Cycling conditions were followed as per manufacturer's protocol and subsequently purified with BigDye Xterminator Purification Kit (ThermoFisher Scientific) before analysis using $A B I$ 3130xl Genetic Analyzer (Applied Biosystems). Sequences were assembled using Vector NTI Advance 11 software (ThermoFisher Scientific) and aligned using the National Center for Biotechnology Information (NCBI) Basic Local Alignment Search Tool (BLAST) for species identification. As $c p b$ and hsp70 give longer assembled sequences of greater than $1,000 \mathrm{bp}$, for consistency with the analysis of these two regions, all sequences of these two amplicons were trimmed at the same position before BLAST. For $c p b$, sequences were trimmed from nucleotide position (nt) 172 to 1173 of the gene with reference to NCBI GenBank accession\# XM_001562090.1, GQ180933.1, EU289030.1, ${ }^{32-34}$ and for hsp70, from nt 35 to 1351 of the gene with reference to NCBI accession\# FN395040.1, FN395052.1, FN395055.1 $1^{35-37}$ before BLAST analysis. In case SS-BLAST of the its2 amplicon was not determined, single nucleotide polymorphism analysis was performed for speciation. ${ }^{18}$

WGS and bioinformatics analysis. WGS and bioinformatics analysis were conducted as a fee-for-service by the Center for Applied Genomics (The Hospital for Sick Children, Toronto, Canada). WGS was conducted by first preparing a DNA library by Illumina Low input DNA library prep kit, then sequenced in Illumina HiSeq 2500 with V4 chemistry paired end sequencing of $2 \times 126$ bases. Cutadapt was used to trim low quality bases (using quality cutoff: 20 ) and to trim sequencing adapters. Quality and adapter trimming were performed at both (5' and $3^{\prime}$ ) ends. Trimmed reads with length less than 36 bp were filtered out. Trimmed reads for all samples were aligned to human genome (b37 decoy) using GSNAP aligner. Reads mapping to human genome were filtered out, and remaining reads were retained to be processed by next step. Reads were de novo assembled using SPAdes and ABySS and assemblies subsequently merged using Quickmerge. Unique reads from all samples were mapped to the same ATCC strains we genome sequenced and to $\mathrm{NCBI}$ reference genomes: L. braziliensis MHOM/BR/75/M2904 (ASM284v2), L. panamensis MHOM/PA/94/PSC-1 (ASM75516v1), and $L$. peruviana SAMEA2767111 (PAB-4377_V1). ${ }^{38-40}$ Percentage number of reads mapped without any mismatch to the aligning genomes were determined.

Arbitration of species identification, composite reference standard, and statistical analysis. A composite reference analysis of its2, $c p b$, hsp70, and mpi RFLP-SS BLAST was conducted whereby final species identification was assigned if at least two assays supported that final species identification. The composite was used as the reference standard against which WGS results were compared.

Species identification by WGS was based on percentage mapped reads (homology) to the ATCC strains and NCBI reference genomes. A threshold of $70 \%$ homology was used to identify a strain as a unique species (Supplemental Table 1). A unique mono-species identification would be made if reads were $\geq 70 \%$ homology for a particular species, with $\geq 10 \%$ difference between the next closest species. A mixed infection was determined if reads achieved $\geq 70 \%$ homology for the mixed species. Lastly, a hybrid was determined if reads achieved $<70 \%$ homology to the hybrid species. Seventy percent homology was used as the cutoff based on the WGS "Percentage mapped reads to the same species" (Supplemental Table 1). This analysis revealed that the lower percent range for mapping to the reference strains with NCBI reference genomes was $71 \%$; thus, $70 \%$ was selected as the cutoff to determine if samples mapped to certain species.

The sensitivity/accuracy for species identification of the standard molecular assays and WGS was determined by comparing with the composite reference standard, where WGS "true positives" were those corroborated by at least two standard molecular assays, and WGS "false negatives" were those discordant with at least two standard molecular assays. As there were no "true negatives" in this analysis, specificity and negative predictive value were not calculated. Statistical analyses were performed by MEDCALC Diagnostic test evaluation calculator (https://www.medcalc.org/calc/ diagnostic_test.php).

\section{RESULTS}

Standard molecular speciation analysis. The discriminatory level of species identification with standard molecular assays varied for the different loci (Table 1 and Supplemental Table 3). Its1-Haelll RFLP was only able to identify included strains to the Viannia subgenus level. The combination of $c p b$-Taql and both Haelll- and Bccl-based hsp70 RFLP narrowed all ATCC and clinical strains to one species except clinical specimen \#2, which had an overlapping hsp70-Haell banding pattern suggesting a hybrid or mixed infection (Table 2). Different loci of SS BLAST analysis gave different species identities. SS BLAST of the cpb loci for ATCC 50158 L. V. panamensis and clinical specimens \#3, 4, and 5 determined these isolates to be L. V. guyanensis, whereas analysis of its 2 and SS BLAST of $h s p 70$ loci determined them to be L. V. panamensis (Table 2 and Supplemental Table 2). Further sequence analysis of cpb locus with ATCC 50126 L. V. guyanensis, ATCC 50158 L. V. panamensis, L. V. guyanensis (NCBI GenBank accession\# GQ180933.1), and L. V. panamensis (NCBI GenBank accession\# EU289030.1) revealed intraspecies single nucleotide polymorphism heterogeneity of ATCC 50158 L. V. panamensis likening it more so to $L$. $V$. guyanensis rather than $L$. V. panamensis at this particular locus (Figure 1). As opposed to all other samples analyzed by hsp70 -SS BLAST in this study, hsp70 SS BLAST failed to discriminate clinical specimen \#2 to a single species further supporting that this isolate is a hybrid (Table 2 and Supplemental Table 2). Although two mpi endpoint PCRs were included in this study to differentiate $L, V$. peruviana from the other Viannia species, Mpi SS BLAST could not narrow down to a single species level for some strains, 


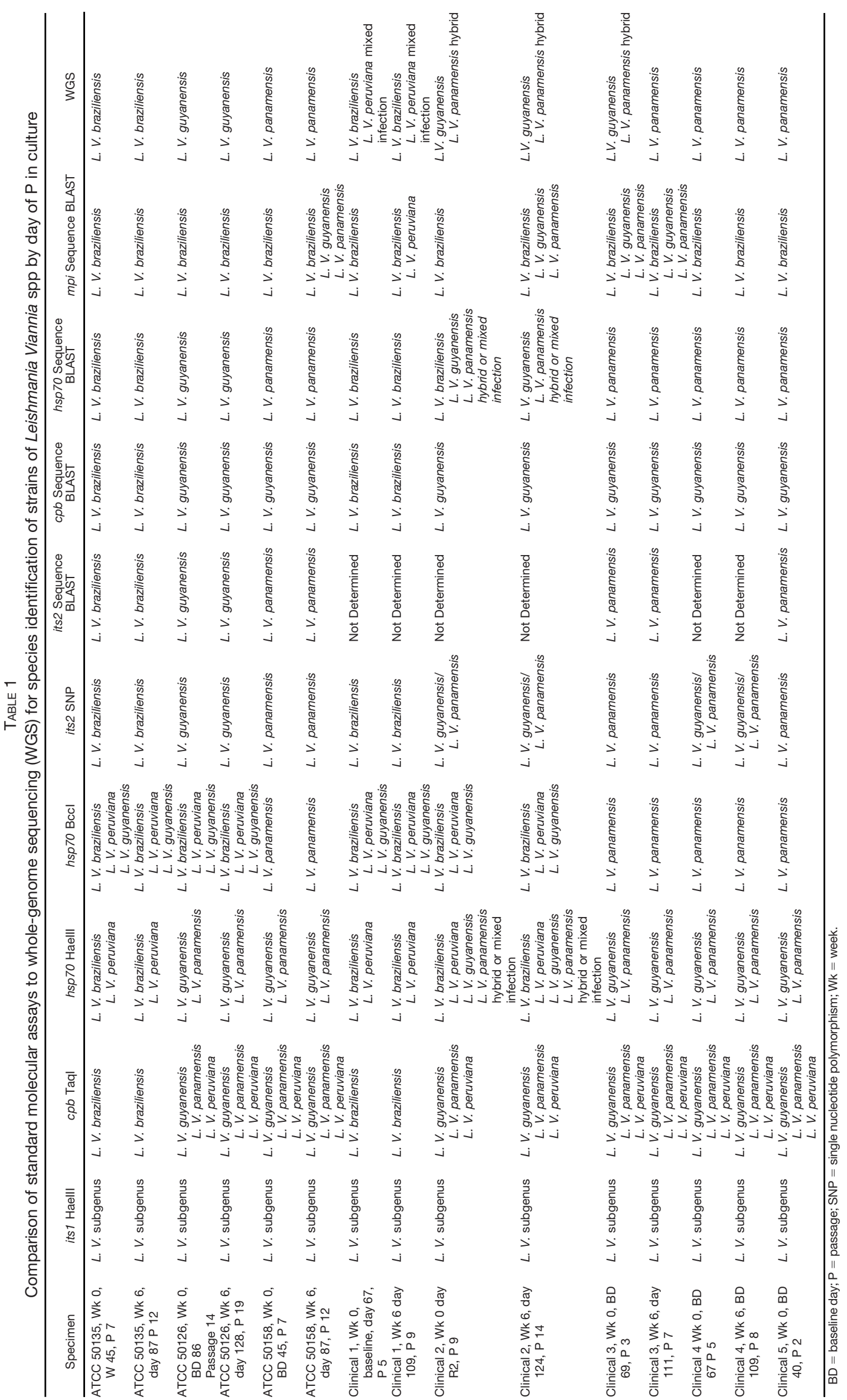


LAU AND OTHERS

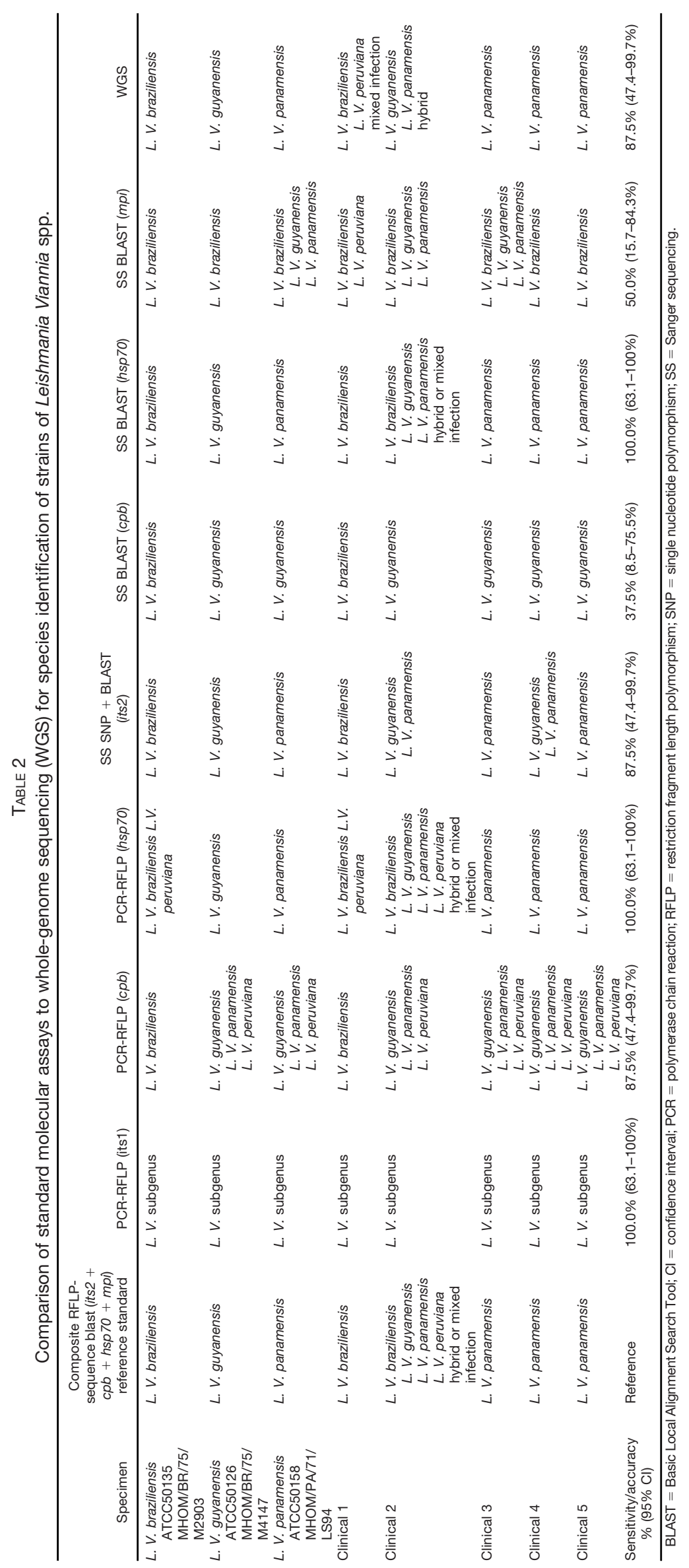




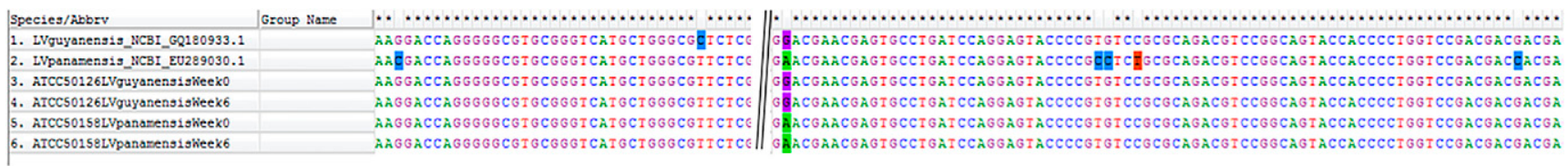

FiguRE 1. Sequence alignment of $c p b$ locus. Alignment of $c p b$ locus displayed intraspecies heterogeneity of ATCC50158 L. V. panamensis strain. This figure appears in color at www.ajtmh.org.

likely due to its low discriminatory level (Table 2 and Supplemental Table 2). Only ATCC $50135^{\mathrm{TM}}$ L. V. braziliensis had a definitive species identification consistent in all RFLP and SS BLAST assays (Table 2 and Supplemental Table 1). Compared with the composite reference, standard molecular assays correctly identified all three ATCC strains and differentiated the clinical isolates into single species within the Viannia subgenus with the exception of clinical specimen \#2, which was likely a hybrid or mixed infection (Table 2).

WGS speciation analysis. Unique reads from WGS of the specific ATCC strains mapped to themselves had an average of $83.2 \%$ (range $79.3-85.3 \%$ ) homology and mapping to the same species of ATCC strains and NCBI reference genomes had an average of $80.8 \%$ (range 71.3-85.3\%) homology (Supplemental Table 1). Intragenome variability of the mapped reads during the 6 weeks of culture had an average of $2.0 \%$ difference (range 0.8-3.0\%) (Supplemental Table 1).

Mapping of WGS reads of ATCC 50135 L. V. braziliensis to itself and to NCBI L. V. braziliensis reference genome at baseline was $79.3 \%$ and $71.3 \%$, respectively, whereas to NCBI L. V. peruviana reference genome was $68.3 \%$, which was less than our threshold of $70 \%$ (Table 3). Upon culture to week 6, homology of all mapped reads increased, with ATCC 50135 L. V. braziliensis WGS reads mapped to itself increased to $82.2 \%, \mathrm{NCBI}$ L. V. braziliensis reference genome to $75.7 \%$ and NCBI L. V. peruviana reference genome to $72.4 \%$. Because the homology to $L$. V. braziliensis was approximately $3 \%$ to $10 \%$ higher than to L. V. peruviana, ATCC 50135 L. V. braziliensis was determined as the final species by WGS analysis.

ATCC 50126 L. V. guyanensis and ATCC 50158 L. V. panamensis strains both mapped to their corresponding species with more than $70 \%$ homology at both time points (Table 3). Although similar to ATCC 50135 L. V. braziliensis, ATCC 50126 L. V. guyanensis also mapped to more than $70 \%$ to $L$. V. panamensis, it had $10 \%$ higher homology to L. V. guyanensis (84.3\% and $85.1 \%$ ) (Table 3). On the basis of WGS analysis, ATCC 50126 L. V. guyanensis and ATCC 50158 L. V. panamensis were determined to be $L$. $V$. guyanensis and $L$. $V$. panamensis respectively.

Clinical sample \#1 showed similar percentages of $>70 \%$ homology to both $L$. V. braziliensis and $L$. V. peruviana at both time points (Table 3). The intragenome culture variability was within range when compared with the ATCC strains suggesting that the homologies between the two time points were consistent. Furthermore, the difference in homology of mapped reads between $L$. $V$. braziliensis and $L$. $V$. peruviana was less than $1 \%$ suggesting that this strain could be a mixed infection of both of these species.

Clinical sample \#2 may represent a signature profile of hybrid species of $L$. V. guyanensis and $L$. V. panamensis as the percentages mapped to both were less than $70 \%$ homology, respectively, and there was only $4 \%$ homology difference between the two species (Table 3).
Clinical sample \#3 resembled a hybrid species of $L$. V. guyanensis and $L$. V. panamensis at baseline (week 0); however, homology to $L$. V. panamensis was $\sim 65 \%$, whereas to $L$. $V$. guyanensis, the homology was $55 \%$, which was $10 \%$ less (Table 3). Further analysis at week 6 confirmed it to be a single L. V. panamensis species with more than $80 \%$ homology (Table 3). The large difference in homology between baseline and week 6 suggests potential selection of strains in culture.

Clinical sample \#4 mapped to be single L. V. panamensis species with over $80 \%$ homology at both time points, whereas clinical sample \#5 mapped to $L$. $V$. panamensis at one time point and had at least $10 \%$ higher homology than to L. V. braziliensis, L. V. guyanensis, or L. V. peruviana (Table 3).

Performance characteristics of standard molecular assays and WGS. Its 1-RFLP, hsp70-RFLP, and hsp70-SS all had 100\% (95\% confidence interval [Cl] 63.1-100\%) accuracy (Table 2). Cpb and mpi SS failed to provide an accurate species identification with only $37.5 \%$ (95\% Cl 8.5-75.5\%) and $50 \%(95 \% \mathrm{Cl}$ 15.7-84.3\%) sensitivity, respectively. WGS had an accuracy of $87.5 \%$ (95\% Cl 47.4-99.7\%). Standard molecular analysis of clinical specimen \#1 revealed it to be a mono L. V. braziliensis species, however, WGS suggested that it was a mixed infection (Table 3). This specimen lowered the accuracy of WGS, however, given the robust analysis of the whole genome in WGS compared with only a small fraction of DNA in the standard molecular assays, WGS provided a higher confidence with the true identity of this specimen being a $L$. V. braziliensis and $L$. V. peruviana mixed infection.

\section{DISCUSSION}

Causative species identification in New World tegumentary leishmaniasis is critical to optimal patient treatment allocation, prognostication, and risk stratification. ${ }^{3}$ However, the strains correlated to the most complex, recalcitrant, and relapsing infections in Latin America are those that are genetically similar members of the Leishmania Viannia subgenus, the discrimination of which has proven challenging for even major diagnostic reference laboratories. ${ }^{14,20,27}$ Thus, more sophisticated and innovative diagnostic platforms, and all the efficiencies that such interventions entail, for the true allocation of species in such tegumentary leishmaniasis infections are needed. We have herein demonstrated the ability of WGS to aid in the species identification and particularly differentiation of mixed and hybrid leishmaniasis infections from mono-infection in the Viannia subgenus using ATCC and NCBI reference strains. WGS's performance is at least comparable and possibly superior to that of current standard molecular diagnostics, with a simpler laboratory workflow algorithm. Instead of sequentially performing PCR, RFLP, and sequencing analyses on multiple conventional molecular targets, which can take up to 2 weeks to conclude, WGS offers a single analytic intervention, and the potential for high-throughput once an efficient 


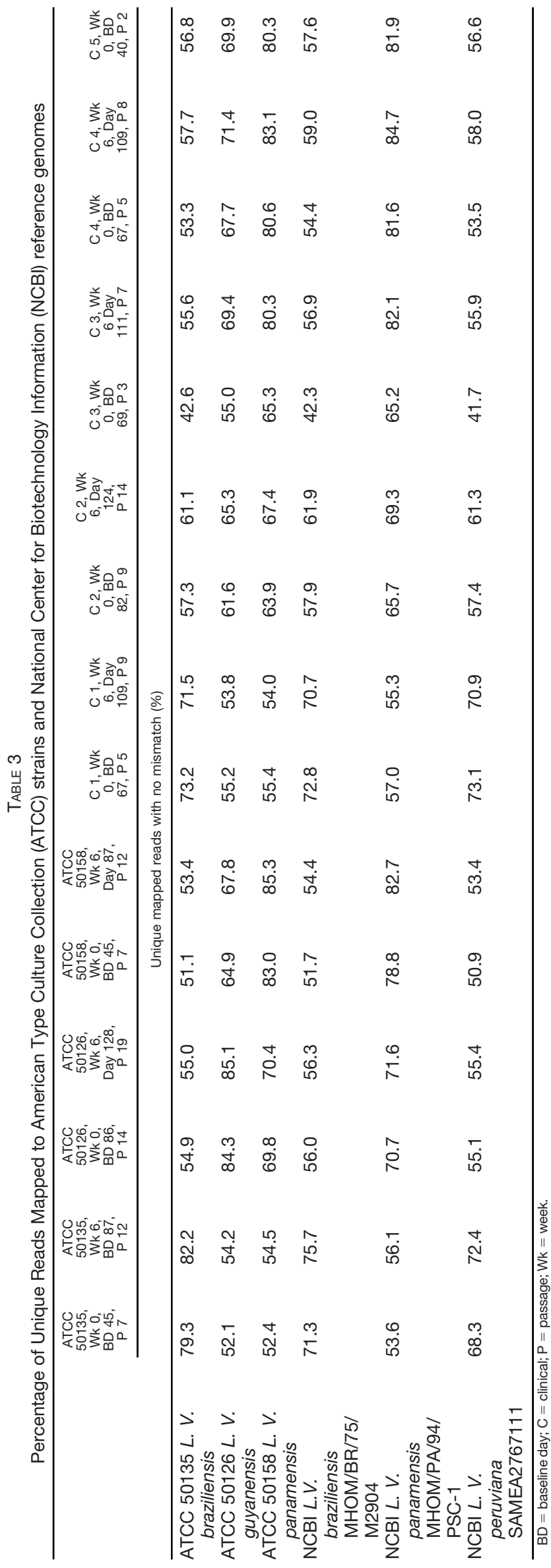

workflow and bioinformatic pipeline is established. Our study required $5 \mu \mathrm{g}$ of DNA for WGS after discussing our objectives with the sequencing and bioinformatics team at The Center for Applied Genomics, a world-renowned next generation sequencing and bioinformatics center. With advancement of WGS technologies and the knowledge we gained from this study, less DNA will be required for further studies and the potential to perform directly on primary clinical specimen. As such, WGS could offer laboratories with more accurate species identification timely for physicians to more appropriately allocate patients to systemic versus local therapy at the time of clinical decision-making. ${ }^{3}$ Present molecular species identification approaches work well for tegumentary leishmaniasis infections that are acquired in areas where only one or two species are known to circulate, or in situations where lesions are simple and unlikely to lead to downstream mucosal involvement (e.g., Old World cutaneous leishmaniasis from counties such as Afghanistan where L. tropica is a common causative species). Under those circumstances, final species identification need not be rendered to deliver appropriate treatment to the patient. Treatment allocation and risk stratification for New World tegumentary leishmaniasis, on the other hand, proves much more challenging, particularly in leishmaniasis from Peru, for example, where multiple species within the Viannia subgenus co-circulate and lead to both cutaneous and mucosal disease. ${ }^{1,2,6,7,10}$ In such scenarios, sequences at standard molecular targets, such as its2, for example, can differ within species by only a single nucleotide, necessitating a multipronged, very granular, labor intensive, and timeconsuming approach to species allocation. WGS has the potential to supplant such approaches and provide clinicians with more rapid, precise, and accurate species identification results, analyzing the whole genome, rather than specific genes, as our molecular analysis has shown, can provide mixed results.

There is enormous genetic diversity within the Viannia subgenus in the New World, owing to markedly high levels of clonality and recombination. ${ }^{41}$ Traditionally, a number of molecular targets are used to interrogate the identity of strains within the Viannia subgenus. Hsp70 RFLP-SS BLAST assays are viewed as the most accurate standard molecular assays for species identification in the Viannia sub-genus. L. V. braziliensis, L. V. guyanensis, L. V. panamensis, L. V. peruviana, and L. V. lainsoni hsp70 sequences are readily available in the NCBI database. Cpb SS BLAST analysis is another approach to Viannia strain interrogation, however, as demonstrated herein, this strategy likely misidentified L. V. panamensis ATCC50158 (MHOM/PA/71/LS94) as L. V. guyanensis, and similarly did so for clinical specimens 3, 4, and 5, likening them more to L. V. guyanensis rather than L. V. panamensis. Mpi PCR can only discriminate $L$. $V$. peruviana from other members of the Viannia subgenus and perhaps is able to identify hybrid infections. However, this assay must be complemented with SS because the primer set that discriminates $L$. V. peruviana from other Viannia strains varies only by a singular nucleotide on the 3 ' end, leading to assay cross-reactivity.

Use of the its1-HaellI PCR-RFLP assay is limited to differentiation of species in Leishmania Viannia subgenus from members of the Leishmania subgenus. The its2 SS assay is limited to detection of L. V. braziliensis, L. V. guyanensis, and L. V. panamensis when using the SS BLAST platform. There is no its2 sequence available in GenBank for $L$. V. peruviana and 
only a partial sequence for $L$. V. lainsoni. Furthermore, its2 has multiple repeating DNA satellite regions, which can make SS technically challenging, resulting in short reads. The combined hsp70-Haell and $h s p 70-B c c l$ PCR-RFLP analyses allow us to differentiate out $L$. V. guyanensis, L. V. lainsoni, and L. V. panamensis from the other Viannia species. The addition of yet another restriction enzyme, Rsal, to the $h s p 70$ PCR-RFLP algorithm can also help to resolve $L$. $V$. peruviana from $L$. $V$. braziliensis, in particular, as elegantly described by Montalvo et al. previously, ${ }^{42}$ underscoring the need to balance exhaustive and comprehensive algorithms with the practical operationalization of rapid and efficient species identification. Although our data demonstrated that PCR-RFLP analysis may provide a clearer picture of hybrid or mixed infections compared with SS alone, the interpretation of these assays still requires a collective interpretation, rather than reliance on a single platform approach.

The necessity for standardization of molecular diagnostics in tegumentary leishmaniasis is made clear by Van der Auwera and colleagues, who compared species identification results among 16 European facilities. ${ }^{27}$ Similar to our findings, in the absence of a superior analytic method (e.g., WGS), many other studies have demonstrated that $h s p 70$ SS remains the top choice for species identification methodology. ${ }^{17,27,43}$ However, the reliance solely on hsp70 can also misallocate closely related Viannia species, necessitating clear communication with clinical teams informing them of the chances of error, especially where treatment is species- or complexdependent, as illuminated previously. ${ }^{27}$

Of particular interest is the potential use of WGS to help parse mixed and hybrid infections of tegumentary leishmaniasis from monoinfections with closely related species that yield mixed results on the most common standard PCR-RFLP or SS approaches, as illustrated by clinical specimens \#1 and \#2 in this study. In those specimens, no single or composite standard molecular approach was sufficient to definitively assign mono, hybrid, or mixed infection status, highlighting the need for an expanded repertoire of assays or innovative tools altogether. Clinically, this is an important issue, as we have previously demonstrated that hybrid or mixed infections are overrepresented among those patients with severe forms of American tegumentary leishmaniasis, and further have very high parasite loads in their specimens. ${ }^{10}$ Given the lack of sophistication of molecular epidemiology of Latin American strains of Viannia to date, we have limited data to support one therapeutic approach over another in such scenarios, but from the data that do exist, we believe that automatic allocation to systemic therapy for such hybrid/mixed infections is warranted. $^{10}$

In addition to having diagnostic implications particularly for Latin American strains of tegumentary leishmaniasis, WGS may also help disentangle the challenging issue of treatment failure and drug resistance. Ubeda and colleagues have shown that stochastic rearrangement of repeated sequences and the selection of copy number variants within specific loci is a novel technique by which Leishmania is able to react to adaptive environments (such as drug-induced pressures). ${ }^{30}$ This can all contribute to an increased ability to evolve drug resistance via competent species hybridization, among many other mechanisms. In L. major, WGS has already been used to pinpoint novel mutation biomarkers of miltefosine resistance. ${ }^{44}$ Moreover, Teixeira and colleagues have reported that the whole genomes of symptomatic $L$. infantum in infected patients have a notably higher level of genetic diversity than those of asymptomatically presenting individuals. ${ }^{45}$ Using WGS for $L$. donovani, Downing and colleagues have contributed new knowledge to both the population structure and potential mechanisms of drug resistance in strains of Leishmania. ${ }^{46}$ The role of sexual reproduction in the establishment of hybrid species of Leishmania has become increasingly important in the context of evolution and drug resistance. ${ }^{47}$ Additionally, WGS in Leishmania donovani has been shown to have the potential to provide reliable and novel vaccine candidate biomarkers via investigation of virulence attenuationassociated genes. ${ }^{48}$ Ultimately, WGS for species identification within the Viannia sub-genus can serve as a powerful tool to study large-scale geographic diversification, evolution, and population-level drug resistance biomarkers.

This study and its associated interpretations are not without limitations. It is well known that the usefulness of SS is dependent on the breadth of the NCBI database, and the accuracy of the correlation of the sequence and species submitted. In our case of the $c p b$ loci, we identified $L$. V. panamensis strains with sequence heterogeneity not captured in NCBI GenBank which falsely mis-identified to $L$. $V$. guyanensis. This phenomenon is not isolated to parasitology: in mycology, as many as $20 \%$ of fungal sequences deposited to GenBank can be incorrectly annotated. ${ }^{49}$ We examined eight strains of the Leishmania Viannia subgenus due to budget limitations. It is possible that our conclusions may have differed with a study enrolling a larger number of strains or an expanded repertoire of restriction enzymes, such as Rsal, and assays. Use of WGS in this study, required pure culture and a large DNA content. Currently, with the constant advancement of sequencing technology, DNA content requirement has significantly lowered to pg of DNA and potentially performed directly on clinical specimen. High cost of WGS can make it inaccessible to resource-limited settings, not to mention that bioinformatics expertise is required to accurately analyze sequences. Current WGS (including bioinformatics cost) is approximately USD $\$ 500$ persample, whereas the standard molecular diagnostics cost about USD $\$ 30$ per sample. With the constantly diminishing cost of WGS over the past decade, it is certainly plausible that the future may bring greater accessibility of the technology and associated expertise.

The ability to discriminate amongst the Viannia strain infections in standard Leishmania diagnostics is an arduous and often time-consuming process that requires multiple molecular assays to arrive at an often-imprecise result. WGS may be a useful species identification platform that is comparable to our standard composite PCR-RFLP and SS analyses. More data are required to make a definitive conclusion about the role of WGS in Leishmania diagnostics. Cost continues to limit access to WGS for Leishmania species identification, with a 17-fold higher cost of WGS on a per sample basis compared with PCR-RFLP and SS algorithms. When further optimized, using WGS for species identification may greatly reduce turnaround time as the need for complicated stepwise algorithms with multipronged molecular diagnostics is eliminated. ${ }^{50} \mathrm{Hav}-$ ing a singular assay, for a singular specimen, through one technological pipeline has its own inherent logistical benefits, unmet by the labor-intensive, intricate, and imprecise stepwise algorithms in current use. ${ }^{50}$ 
Received March 4, 2021. Accepted for publication May 4, 2021.

Published online July 16, 2021.

Note: Supplemental figure and tables appear at www.ajtmh.org.

Acknowledgments: We thank Sergio Pereira, Bhooma Thiruvahindrapuram, and Gaganjot Kaur for their genome sequencing and bioinformatics advice and analysis.

Disclosure: This study was approved by the Ethics Research Board of Public Health Ontario.

Financial support: This work was funded by Public Health Ontario via a Project Initiation Fund.

Authors' addresses: Rachel Lau, Public Health Ontario Laboratory, Toronto, Ontario, Canada, E-mail: rachel.lau@oahpp.ca. Avinash N. Mukkala, Institute of Medical Science, University of Toronto, Toronto, Ontario, Canada, E-mail: avinash.mukkala@mail.utoronto. ca. Ruwandi Kariyawasam, University of Alberta, Edmonton, Alberta, Canada, E-mail: ruwandi@ualberta.ca. Shareese Clarke, Faculty of Nursing, University of Toronto, Toronto, Ontario, Canada, E-mail: shareese.clarke@mail.utoronto.ca. Braulio M. Valencia, Kirby Institute, New South Wales, Australia, E-mail: braulio.valencia@upch. pe. Alejandro Llanos-Cuentas, Universidad Peruana Cayetano Heredia, Lima, Peru, E-mail: alejandro.llanos.c@upch.pe. Andrea K. Boggild, Institute of Medical Science and the Department of Medicine, University of Toronto, Toronto, Ontario, Canada, E-mail: andrea.boggild@utoronto.ca,

\section{REFERENCES}

1. Llanos-Cuentas A et al., 2008. Clinical and parasite species risk factors for pentavalent antimonial treatment failure in cutaneous leishmaniasis in Peru. Clin Infect Dis 46: 223-231.

2. Arevalo $J$ et al., 2007. Influence of Leishmania (Viannia) species on the response to antimonial treatment in patients with American tegumentary leishmaniasis. J Infect Dis 195: 1846-1851.

3. Aronson $\mathrm{N}$ et al., 2016. Diagnosis and treatment of leishmaniasis: clinical practice guidelines by the Infectious Diseases Society of America (IDSA) and the American Society of Tropical Medicine and Hygiene (ASTMH).

4. Reithinger R, Dujardin J-C, Louzir H, Pirmez C, Alexander B, Brooker S, 2007. Cutaneous leishmaniasis. Lancet Infect Dis 7: 581-596.

5. Boggild AK et al., 2010. Clinical and demographic stratification of test performance: a pooled analysis of five laboratory diagnostic methods for American cutaneous leishmaniasis. Am J Trop Med Hyg 83: 345-350.

6. Boggild AK et al., 2011. Diagnostic performance of filter paper lesion impression PCR for secondarily infected ulcers and nonulcerative lesions caused by cutaneous leishmaniasis. J Clin Microbiol 49: 1097-1100.

7. Boggild AK et al., 2010. Detection and species identification of Leishmania DNA from filter paper lesion impressions for patients with American cutaneous leishmaniasis. Clin Infect Dis 50: e1-e6.

8. Boggild AK et al., 2011. Non-invasive cytology brush PCR diagnostic testing in mucosal leishmaniasis: superior performance to conventional biopsy with histopathology. PLoS One 6: e26395.

9. Akhoundi $\mathrm{M}$ et al., 2017. Leishmania infections: molecular targets and diagnosis. Mol Aspects Med 57: 1-29.

10. Valencia BM et al., 2016. Quantitative kinetoplast DNA assessment during treatment of mucosal leishmaniasis as a potential biomarker of outcome: a pilot study. Am J Trop Med Hyg 94: 107-113.

11. Kotb Elmahallawy E et al., 2014. Diagnosis of leishmaniasis. $J$ Infect Dev Ctries 8: 961-972.

12. World Health Organization, 2010. Expert Committee on the Control of the Leishmaniases \& World Health Organization. Control of the leishmaniases: report of a meeting of the WHO Expert Committee on the Control of Leishmaniases, Geneva, 22-26 March 2010. Available at: https://apps.who.int/iris/handle/ $10665 / 44412$.
13. Valencia BM et al., 2012. Non-invasive cytology brush PCR for the diagnosis and causative species identification of American cutaneous leishmaniasis in Peru. PLoS One 7: e49738.

14. Van der Auwera G, Dujardin J-C, 2015. Species typing in dermal leishmaniasis. Clin Microbiol Rev 28: 265-294.

15. Montalvo AM, Fraga J, Maes I, Dujardin J-C, Van der Auwera G, 2012. Three new sensitive and specific heat-shock protein 70 PCRs for global Leishmania species identification. Eur J Clin Microbiol Infect Dis 31: 1453-1461.

16. Veland $\mathrm{N}$ et al., 2013. Simultaneous infection with Leishmania (Viannia) braziliensis and L. (V.) lainsoni in a Peruvian patient with cutaneous leishmaniasis. Am J Trop Med Hyg 88: 774777.

17. Fraga $J$ et al., 2012. Accurate and rapid species typing from cutaneous and mucocutaneous leishmaniasis lesions of the New World. Diagn Microbiol Infect Dis 74: 142-150.

18. de Almeida ME, Steurer FJ, Koru O, Herwaldt BL, Pieniazek NJ, da Silva AJ, 2011. Identification of Leishmania spp. by molecular amplification and DNA sequencing analysis of a fragment of rRNA Internal Transcribed Spacer 2. J Clin Microbiol 49: 3143-3149.

19. Mahdy MAK et al., 2010. Molecular characterization of Leishmania species isolated from cutaneous leishmaniasis in Yemen. PLoS One 5: e12879.

20. Rotureau B et al., 2006. Use of PCR-restriction fragment length polymorphism analysis to identify the main New World Leishmania species and analyze their taxonomic properties and polymorphism by application of the assay to clinical samples. J Clin Microbiol 44: 459-467.

21. Schönian G et al., 2003. PCR diagnosis and characterization of Leishmania in local and imported clinical samples. Diagn Microbiol Infect Dis 47: 349-358.

22. Victoir $\mathrm{K}$ et al., 2003. Direct identification of Leishmania species in biopsies from patients with American tegumentary leishmaniasis. Trans R Soc Trop Med Hyg 97: 80-87.

23. Zhang W-W et al., 2006. Development of a genetic assay to distinguish between Leishmania viannia species on the basis of isoenzyme differences. Clin Infect Dis 42: 801-809.

24. Garcia AL et al., 2005. American tegumentary leishmaniasis: antigen-gene polymorphism, taxonomy and clinical pleomorphism. Infect Genet Evol 5: 109-116.

25. Garcia L et al., 2004. Culture-independent species typing of neotropical Leishmania for clinical validation of a PCR-based assay targeting heat shock protein 70 genes. $J$ Clin Microbiol 42: 2294-2297.

26. Tsokana CN, Athanasiou LV, Valiakos G, Spyrou V, Manolakou K, Billinis C, 2014. Molecular Diagnosis of Leishmaniasis, Species Identification and Phylogenetic Analysis. LeishmaniasisTrends in Epidemiology, Diagnosis and Treatment. InTech.

27. Van der Auwera G et al., 2016. Comparison of Leishmania typing results obtained from 16 European clinical laboratories in 2014. Euro Surveill 21: 30418

28. Reithinger R, Dujardin J-C, 2007. Molecular diagnosis of leishmaniasis: current status and future applications. J Clin Microbiol 45: 21-25.

29. Cassagne $C$ et al., 2014. Identification of Leishmania at the species level with matrix-assisted laser desorption ionization time-of-flight mass spectrometry. Clin Microbiol Infect 20: $551-557$.

30. Ubeda J-M et al., 2014, Genome-wide stochastic adaptive DNA amplification at direct and inverted DNA repeats in the parasite Leishmania. PLoS Biol. 12: e1001868.

31. Quispe Tintaya KW, Ying X, Dedet J, Rijal S, De Bolle X, Dujardin J, 2004. Antigen genes for molecular epidemiology of leishmaniasis: polymorphism of cysteine proteinase $B$ and surface metalloprotease glycoprotein 63 in the Leishmania donovani complex. J Infect Dis 189: 1035-1043.

32. NCBI GenBank xm_001562090.1. Available at: https://www.ncbi. nlm.nih.gov/search/all/?term=xm_001562090.1. Accessed July 3, 2019.

33. NCBI GenBank GQ180933, 1. Available at: https://www.ncbi.nlm. nih.gov/search/all/?term=GQ180933.1. Accessed July 3, 2019.

34. NCBI GenBank EU289030, 1. Available at: https://www.ncbi.nlm. nih.gov/nuccore/EU289030.1. Accessed July 3, 2019. 
35. NCBI GenBank FN395040, 1. Available at: https://www.ncbi.nlm. nih.gov/nuccore/FN395040.1. Accessed July 3, 2019.

36. NCBI GenBank FN395052, 1. Available at: https://www.ncbi.nIm. nih.gov/nuccore/FN395052.1. Accessed July 3, 2019.

37. NCBI GenBank FN395055, 1. Available at: https://www.ncbi.nIm. nih.gov/nuccore/FN395055.1. Accessed July 3, 2019.

38. Leishmania peruviana (ID 40809) - Genome - NCBI. Available at: https://www.ncbi.nlm.nih.gov/genome/?term=L.+peruviana. Accessed July 3, 2019.

39. Leishmania panamensis (ID 13991) - Genome - NCBI. Available at: https://www.ncbi.nlm.nih.gov/genome/ ?term=MHOM\%2FPA\%2F94\%2FPSC-1. Accessed July 3, 2019.

40. Leishmania braziliensis (ID 718) - Genome - NCBI. Available at: https://www.ncbi.nlm.nih.gov/genome/?term=L. +braziliensis+MHOM\%2FBR\%2F75\%2FM2904.

41. Kuhls $\mathrm{K}$ et al., 2013. Population structure and evidence for both clonality and recombination among Brazilian strains of the subgenus Leishmania (Viannia). PLoS Negl Trop Dis 7: e2490.

42. Montalvo AM, Fraga J, Monzote L, Montano I, De Doncker S, Dujardin JC, Van der Auwera G, 2010. Heat-shock protein 70 PCR-RFLP: a universal simple tool for Leishmania species discrimination in the New and Old World. Parasitology 137: 11591168.

43. Cruz-Barrera ML, Ovalle-Bracho C, Ortegon-Vergara V, PérezFranco JE, Echeverry MC, 2015. Improving Leishmania species identification in different types of samples from cutaneous lesions. J Clin Microbiol 53: 1339-1341.

44. Coelho AC, Boisvert S, Mukherjee A, Leprohon P, Corbeil J, Ouellette $M, 2012$. Multiple mutations in heterogeneous miltefosine- resistant Leishmania major population as determined by whole genome sequencing. PLoS Negl Trop Dis 6: e1512.

45. Teixeira DG et al., 2017. Comparative analyses of whole genome sequences of Leishmania infantum isolates from humans and dogs in northeastern Brazil. Int J Parasitol 47: 655-665.

46. Downing T et al., 2011. Whole genome sequencing of multiple Leishmania donovani clinical isolates provides insights into population structure and mechanisms of drug resistance. Genome Res 21: 2143-2156.

47. Rogers MB et al., 2014. Genomic confirmation of hybridisation and recent inbreeding in a vector-isolated Leishmania population. PLoS Genet. 10: e1004092.

48. Gannavaram S et al., 2016. Modulation of innate immune mechanisms to enhance Leishmania vaccine-induced immunity: role of coinhibitory molecules. Front Immunol 7: 187.

49. Raja HA, Miller AN, Pearce CJ, Oberlies NH, 2017. Fungal identification using molecular tools: a primer for the natural products research community. J Nat Prod 80: 756-770.

50. Veland $\mathrm{N}$ et al., 2012. Leishmania (Viannia) species identification on clinical samples from cutaneous leishmaniasis patients in Peru: assessment of a molecular stepwise approach. J Clin Microbiol 50: 495-498.

51. Cupolillo E, Grimaldi Júnior G, Momen H, Beverley SM, 1995. Intergenic region typing (IRT): a rapid molecular approach to the characterization and evolution of Leishmania. Mol Biochem Parasitol 73: 145-155.

52. Montalvo AM et al., 2008. PCR-RFLP and RAPD for typing neotropical Leishmania. Biomedica 28: 597-606. Available at: http://www.ncbi.nlm.nih.gov/pubmed/19462565. Accessed July 3, 2019. 